\title{
THERMAL PROPERTIES OF TPD-BASED ORGANIC GLASSES
}

\author{
RICCARDO DETTORI, (*) LUCIANO COLOMBO $(* *)$
}

SUNTO. - I vetri sono sistemi di materia condensata con proprietà fisiche intermedie tra quelle dei liquidi e quelle dei solidi. La loro struttura atomistica è determinata sia dalla loro storia termica sia dai metodi di preparazione. Da un punto di vista formale ciò corrisponde a un'iper-superficie di energia configurazionale di topologia molto complessa, caratterizzata da molti diversi bacini che differiscono per forma, estensione e profondità. Il sistema può essere intrappolato in ciascuno di essi, assumendo proprietà fisiche significativamente diverse. È stato dimostrato sperimentalmente che è possibile ottenere uno stato vetroso per deposizione di molecole organiche da fase vapore su un substrato solido. In questo caso la fisica del vetro risultante è ancora più complessa perché entrano in gioco fattori addizionali, quale ad esempio l'anisotropia della sottostante struttura molecolare. Abbiamo generato al computer un insieme di campioni di vetri organici costituiti da molecole TPD, mimando il processo di deposizione da fase vapore e ne abbiamo calcolato le proprietà termiche mediante tecniche di simulazione atomistica. In particolare, abbiamo elaborato un modello molto accurato capace (i) di spiegare le risultanze sperimentali indicanti un carattere fortemente anisotropo nella trasmissione di calore e (ii) di predire l'andamento in temperatura del calore specifico di questi vetri. In particolare, riportiamo nel regime quantistico di bassissime temperature l'evidenza di un'interessante anomalia rispetto ai sistemi cristallini.

$* * *$

ABSTRACT. - Glassy materials are condensed matter systems showing physical properties in between solids and liquids and retaining information about the thermal history they have been subjected to and the way they have been prepared. Formally, this implies that their configurational energy landscape is a complex multi-dimensional surface, showing quite a few basins with different depths, widths, and shapes: the system can be trapped in any of them, assuming very unlike physical properties. Recently, it has been demonstrated experimentally that a glassy system can be grown by physical vapor deposition of organic molecules on a substrate. The physics of such organic glasses is

(*) Dipartimento di Fisica dell'Università di Cagliari, Italia.

${ }^{(* *)}$ Istituto Lombardo Accademia di Scienze e Lettere, Milano. Dipartimento di Fisica dell’Università di Cagliari, Italia. E-mail: luciano.colombo@unica.it 
enriched by a new feature, namely: the anisotropic molecular structure of the basic building blocks used to assemble the film. TPD-based organic glasses have been generated by atomistic simulations that mimic vapor deposition and their thermal properties have been accordingly calculated. Simulations generate a rational phenomenology, providing robust evidence that heat transfer is not isotropic but, rather, correlated to an inherent molecular property, namely the axial structure of the TPD molecule. Furthermore, we present the first theoretical prediction of the specific heat trend versus temperature, showing in the quantum regime an intriguing anomaly with respect to crystalline systems.

\section{INTRODUCTION}

Glasses can be looked at as fluid systems that lost their ability to flow: in fact, from a structural point of view a glass is practically indistinguishable from the liquid phase prior to its formation. Glassy materials combine the disordered structure of a liquid with the physical properties of a solid: this unique combination makes them really interesting under many respects.

Upon cooling below the freezing temperature (hereafter referred to as $\mathrm{T}_{\mathrm{m}}$ ) molecular motion slows down. If the liquid is cooled sufficiently fast, crystallization can be avoided and "supercooled liquid" regime is achieved, that is a metastable phase is formed in which molecules will rearrange so slowly that they cannot adequately sample configurations in the available time allowed by the cooling rate [1,2]. This characteristic relaxation time can increase by several orders of magnitude (i.e. up to $10^{2}-10^{3}$ seconds), while the rate of change of volume or enthalpy with respect to temperature decreases abruptly (but continuously) to a value comparable to that of a crystalline solid. The temperature at which these changes occur is referred to as the glass transition temperature (hereafter referred to as $\mathrm{T}_{\mathrm{g}}$ ). Typical laboratory cooling rates vary from 0.1 to $100 \mathrm{~K} / \mathrm{min}$, considerably slower than the rates achievable in computer simulations. The slower a liquid is cooled down, the longer is the time available for configurational sampling at each temperature and, hence, the colder turns out to lie the state reached by the system before falling out of liquid-state equilibrium. Consequently, $\mathrm{T}_{\mathrm{g}}$ increases with cooling rate $[3,4]$. The properties of a glass, therefore, depend on the process by which it is formed. Furthermore, glassy systems can be described in terms of a potential energy landscape, their thermodynamics and kinetics being controlled 
by the minima and barriers on the landscape, respectively [1-5]. Many important issues could be addressed if liquids or glasses with very low energies could be generated [6-8]. For example, it might be possible to definitively understand the Kauzmann entropy crisis [9-11].

Organic glasses represent a special kind of vitreous systems, interesting for both basic science and technological applications [12]. Their use in electronics as organic light-emitting devices (OLEDs) [13] is no longer a lab curiosity but, rather, a mature technology for high-performance displays $[13,14]$. On the other hand, solid-state lightning applications requiring high brightness, are still out-of-reach due to the poor thermal stability of the organic materials: as a matter of fact, thermal stress and degradation [15], together with the fact that both the luminescence and the lifetime of OLEDs decreases when operated at high temperatures [16], are widely reported facts. In this respect, an appropriate understanding of their thermal properties may help designing materials with power dissipation characteristics tailored to minimize heat accumulation in OLEDs $[17,18]$ or to reduce heat flow while increasing charge transport. In a different perspective, their semiconductor nature and their inherent low thermal conductivities make them suitable candidates for thermoelectric generators [19-22]. Most of the previous studies in this direction have been addressed to polymer-based devices, rather than to small-molecule organic semiconductors [23].

Recently, it has been reported that vapor deposition can bypass the kinetic restrictions typically observed during glass formation and, thus, generate a new class of glassy materials with extraordinary energetic and kinetic stability, as well as unusually high densities. In particular, such achievements have been demonstrated for two organic glass formers: 1,3-bis-(1-naphthyl)-5-(2-naphthyl)benzene (TNB) $\left(\mathrm{T}_{\mathrm{g}}=347\right.$ $\mathrm{K})$ and indomethacin (IMC) $\left(\mathrm{T}_{\mathrm{g}}=315 \mathrm{~K}\right)$ [24]. Physical vapor deposition has been also shown to be a suitable tool for tailoring the properties of the deposited layers [25-32]. When the deposition conditions (basically, the substrate temperature and the growth rate) are properly set, then glasses with exceptional thermodynamic and kinetic stability $[25,27,28,33]$, high densities [26, 34-36], low heat capacities [29, 37], low water uptake [38] or high moduli [39] can be obtained. These systems, named as "ultrastable", are currently gaining attention within the glass community, and a recent report demonstrates the improved packing of these glasses can yield to outstanding improvements in OLEDs efficiency [40]. 
An interesting feature of some vapor-deposited organic glasses is that molecules can arrange with a preferential spatial orientation, differing from the random distribution typical of a isotropic glass. Recent studies addressed this issue and its influence on the efficiency of OLEDs [41]. The existence of molecular packing anisotropy in vapordeposited organic semiconductor thin film glasses was first identified by Lin et al. [42]. Yokoyama and coworkers [43, 44] studied the degree of orientation depending on the molecular aspect ratio of the molecule and the deposition conditions. Dalal et al. [25] performed dichroism and birefringence measurements on several organic semiconductors and proposed the ratio between the deposition temperature and the glass transition temperature, $\mathrm{T}_{\mathrm{dep}} / \mathrm{T}_{\mathrm{g}}$, to be the primary parameter affecting the molecular orientation. In particular, it has been shown that the lower the substrate temperature during growth, the higher the tendency towards horizontal orientation. This tunable molecular orientation provides new opportunities to tailor the electrical, thermal and optical properties of the glassy materials. Many investigations have been also focused on the electronic transport properties of organic glasses and crystals, since this is a key parameter for the use of such materials in optoelectronic devices [45].

On the other hand, thermal properties of small-molecule organic glasses remain largely unexplored: in fact, just few studies are reported on heat transport features [46, 47]. In general, it is well known that structural disorder largely affects thermal conductivity: for instance, in silicon its room-temperature value varies from $150 \mathrm{~W}$ $\mathrm{m}^{-1} \mathrm{~K}^{-1}$ in a crystalline sample to $\sim 1.4 \mathrm{~W} \mathrm{~m}^{-1} \mathrm{~K}^{-1}$ in an amorphous one $[48,49]$. This low value is understood in the framework of the minimum thermal conductivity model, where only atomic vibrations with mean free paths of the order of the interatomic distance mainly contribute to heat transport [50]. By the very nature or organic materials, the van der Waals (vdW) interactions among molecules are expected to have a remarkable effect on heat propagation, while structural disorder is expected to play a comparatively less dramatic effect on the thermal conductivity compared to their inorganic counterparts. It is however hard to go beyond the above qualitative expectations, since our understanding of heat conduction in organic glasses is still limited by the largely incomplete knowledge about the actual mechanisms ruling over thermal energy exchange and how the glass atomic-scale morphology affects transport. In particular, available measurements 
on thin-film organic crystals lack a proper understanding of how anisotropy may affect thermal transport along and perpendicular to the molecular chain. Ac-calorimetry was previously used to extract the thermal diffusivity of rubrene layers [51]. The thermal anisotropy ratio defined as the relative difference between in-plane and throughplane conductivity, $\left(\kappa_{\|}-\kappa_{\perp}\right) / \kappa_{\perp}$ (see Fig. 1), was larger than $100 \%$ indicating poor thermal transport across the phenyl groups of the rubrene molecules. On the contrary measurements on 6, 13- Bis(triisopropylsilylethynyl)pentacene, TIPSpn, show the through-plane thermal diffusivity is larger than the in-plane one due to an excellent $\pi$-orbital overlap [52]. The role of thermal anisotropy has been already addressed in polymeric samples [53] where rubbing or stretching has been used to produce the alignment of the backbone of the polymer along the fiber direction. In this case, the conductivity along the axis of the polymeric chain can be up to 20 times higher than in the perpendicular direction [54].

In this work we report a thorough investigation on the thermal properties of TPD-based organic glasses. In particular, we provide evidence that, by tuning the molecular orientation, the resulting thermal anisotropy ratio can be modified to nearly $16 \%$. This change is mainly driven by the molecular packing anisotropy in the glass. We also prove that thermal transport along the TPD molecule backbone is strongly preferred with respect to the perpendicular direction due to a stronger molecular interaction in the former. Finally, we calculate the heat capacity of the same TPD-based organic glass predicting its trend of variation in the low temperature quantum regime.

\section{Methods}

\subsection{Calculating thermal conductivity}

In the present work we investigated thermal properties of organic glasses by means of two different approaches, both relying on classical molecular dynamics simulations.

In the first case, the thermal conductivity tensor $\kappa_{\lambda \mu}^{\mathrm{GK}}$ of a system of volume $\mathrm{V}$ at temperature $\mathrm{T}$ is calculated, according to the equilibrium Green-Kubo formalism, by evaluating the auto-correlation function of the heat flux $\overrightarrow{\mathbf{J}}(\mathrm{t})$ 


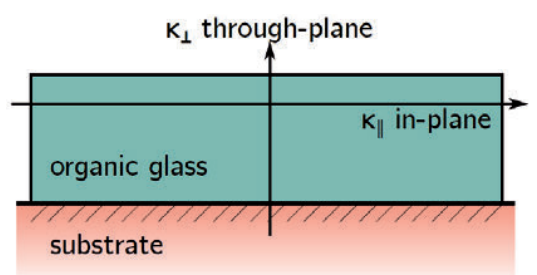

Fig. 1. Schematic representation of a deposited organic glass, reporting the concept of in-plane $\kappa_{\|}$and through-plane $\kappa_{\perp}$ thermal conductivity.

We remark that $\kappa_{\|}$is average value of the thermal conductivities measured along the two directions parallel to the substrate.

$\kappa_{\lambda \mu}^{\mathrm{GK}}(\mathrm{T})=\frac{1}{3 \mathrm{Vk}_{\mathrm{B}} \mathrm{T}^{2}} \lim _{\mathrm{t} \rightarrow \infty} \int_{0}^{\mathrm{t}}\left\langle\mathrm{J}_{\lambda}\left(\mathrm{t}^{\prime}\right) \mathrm{J}_{\mu}(0)\right\rangle_{\mathrm{T}} \mathrm{dt}^{\prime}$

where $\langle\ldots\rangle_{\mathrm{T}}$ is the ensemble average and $\lambda, \mu=\mathrm{x}, \mathrm{y}, \mathrm{z}$. The heat current is evaluated through its virial expression [55]

$\overrightarrow{\mathrm{J}}(\mathrm{t})=\frac{\mathrm{d}}{\mathrm{dt}} \sum_{\mathrm{i}} \overrightarrow{\mathrm{r}}_{\mathrm{i}}(\mathrm{t}) \mathrm{e}_{\mathrm{i}}$

where $e_{i}$ is the per-atom energy (including both the potential and the kinetic conribution) of the $\mathrm{i}$-th particle and $\overrightarrow{\mathrm{r}}_{\mathrm{i}}$ is the corresponding position. The evaluation of $\overrightarrow{\mathbf{J}}(\mathrm{t})$ requires the calculation of several contributions, provided by the kinetic energy, the Coulomb potential energy, and all potential energy terms describing the bond, angle, dihedral, and improper contributions (looped over all the atomic pairs, triplets, and quadruplets).

In order to evaluate the Green-Kubo integral appearing in eq.(1) the system was at first aged for 20 ps in the constant-temperature constant-volume ensemble (Nosé-Hoover thermostats have been used). Then a microcanonical run followed for another 300 ps, correlating the heat current each 5 timesteps. The thermal conductivity was calculated throughout the whole microcanonical run, by considering the average value taken over the last 50 ps of simulation.

In the second case, simulations are performed in the transient regime which stands between a suitable initial non-equilibrium configuration and the final equilibrium state. The Approach-to-Equilibrium Molecular Dynamics (AEMD) method was here adopted [56], based on the solution of the one-dimensional heat equation. More specifically, a step-like temperature profile with an initial offset $T_{1}-T_{2}$ is imposed along, say, the $z$ direction. By imposing the periodic boundary condi- 
tions, the heat equation is readily solved and the most general solution for the time variation of the temperature profile is expressed in terms of Fourier series as

$$
\mathrm{T}(\mathrm{z}, \mathrm{t})=\mathrm{A}_{0}+\sum_{\mathrm{i}}\left[\mathrm{A}_{\mathrm{n}} \cos \left(\alpha_{\mathrm{n}} \mathrm{z}\right)+\mathrm{B}_{\mathrm{n}} \sin \left(\alpha_{\mathrm{n}} \mathrm{z}\right)\right] \mathrm{e}^{-\alpha_{\mathrm{n}}^{2} \overline{\mathrm{\kappa}} \mathrm{t}}
$$

where $\alpha_{n}=2 \pi n / L_{z}, \bar{\kappa}$ is thermal diffusivity of the simulated system, and the coefficients $A_{0}, A_{n}$ and $B_{n}$ depend only on the initial condition. Thus, the analytic solution $\mathrm{T}(\mathrm{z}, \mathrm{t})$ depends on the geometry of the problem, on initial temperature drop, and on thermal diffusivity which is related to thermal conductivity through $\bar{\kappa}=\kappa / \rho c_{v}$. This protocol can be easily implemented in a MD simulation: once that the left and right regions of the simulation cell have been initially thermostatted at temperature $T_{1}$ and $T_{2}$ respectively, the system is then aged in a microcanonical run and the initial step-like temperature profile is progressively smoothed by thermal conduction. It is, therefore, possible to define the average temperatures $T_{1, a v e}$ and $T_{2 \text {,ave }}$ in the two semi-cells, which will vary in time while the system is approaching a uniform temperature (i.e. equilibrium) configuration. During such a transient regime the time-dependent difference in average temperatures $\Delta \mathrm{T}(\mathrm{t})=$ $\mathrm{T}_{1, \text { ave }}-\mathrm{T}_{2, \text { ave }}$ is calculated by exploiting eq.(3) and it displays as

$$
\Delta T(t)=\sum_{n=1}^{\infty} C_{n} e^{-\alpha_{n}^{2} \bar{k} t}
$$

where the coefficients $C_{n}$ include information on the geometry and on the initial conditions

$$
\mathrm{C}_{\mathrm{n}}=\frac{\left(\mathrm{T}_{1}-\mathrm{T}_{2}\right)\left[\cos \left(\frac{\alpha_{\mathrm{n}} \mathrm{L}_{\mathrm{z}}}{2}\right)-1\right]^{2}}{\alpha_{\mathrm{n}}^{2} \mathrm{~L}_{\mathrm{z}}^{2}}
$$

The temperature difference $\Delta \mathrm{T}(\mathrm{t})$ is computed on-the-fly during the NVE run, and the collected data are eventually fitted by the analytic solution given in eq.(4) in order to obtain the system thermal diffusivity $\bar{\kappa}$.

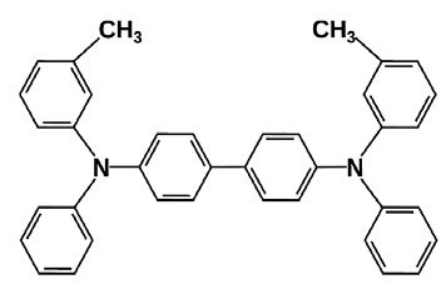

Fig. 2. Schematic representation of a TPD molecule. 


\subsection{The force field}

The TPD molecule, shown in Fig. 2, is modeled according to the CVFF force field [57], where cross-coupling terms between the various bonded objects provide an accurate description of intramolecular interactions. The non-bonding interactions are, in turn, described by a superposition of a Lennard-Jones potential (addressed to describing the vdW contribution) and a Coulomb term as follows

$$
\sum_{\mathrm{i}, \mathrm{j}}\left(\frac{\mathrm{A}}{\mathrm{r}_{\mathrm{ij}}^{2}}-\frac{\mathrm{B}}{\mathrm{r}_{\mathrm{ij}}^{6}}\right)+\sum_{\mathrm{i}, \mathrm{j}} \frac{\mathrm{q}_{\mathrm{i}} \mathrm{q}_{\mathrm{j}}}{\mathrm{r}_{\mathrm{ij}}}
$$

where the sum is performed over all the pairs of non-bonded atoms. The Lennard-Jones term is truncated by a cut-off set at $10.0 \AA$, while a particle-particle particle-mesh solver approach is adopted to solve the electrostatic problem in the reciprocal space. Coulomb interactions are calculated by assuming fixed charges, as previously obtained by fitting the electrostatic potential of an all-electron Hartree-Fock calculation performed with a medium-sized basis set $6-31 G^{*}$. The fitting was performed using the RESP method. [58,59]

Finally, the Tersoff [60] force field is adopted to describe the silicon substrate, giving such bond-order potential also the role of describing the interactions among molecular carbon atoms and silicon atoms in the substrate.

\subsection{Molecular Dynamics simulations}

Molecular dynamics simulations are executed by means of the LAMMPS [61] package.

Equations of motion are integrated according to the velocityVerlet algorithm with a time step of $0.5 \mathrm{fs}$. Time integration is performed on Nosé-Hoover non-Hamiltonian equations for both constant-pressure (NPT) and constant-temperature (NVT) runs, respectively used to reach equilibrium density at a given temperature and to further equilibrate the sample at a given temperature. Thermostatting and barostatting are achieved by a coupling parameter of $50.0 \mathrm{fs}$ and a relaxation time of $500.0 \mathrm{fs}$, respectively.

\subsection{Sample preparation}

In order to generate TPD-based glassy samples, we exploited 
the role of the substrate to enforce anisotropy. A silicon substrate obtained by $20 \mathrm{a}_{0} \times 20 \mathrm{a}_{0} \times 5 \mathrm{a}_{0}$ replicas of the conventional cell was at first realized, replicating the geometry shown in Fig. 1 (here $\mathrm{a}_{0}=5.4305 \AA$ is the equilibrium silicon lattice constant for the adopted Tersoff potential). Next, two kinds of anisotropic TPD films have been realized (hereafter respectively referred to as xy-ISO and ANIS), both are characterized by a preferential in-plane molecular orientation. This feature has been obtained by enforcing the alignment of any molecular axis parallel to the substrate (see Fig. 1).

A first layer of 47 molecules was placed on top of the substrate, followed by a geometry optimization and a low temperature $(\mathrm{T}=1 \mathrm{~K})$ annealing for 100 ps. While the in-plane alignment was totally random in the xy-ISO sample, TPD molecules have been further enforced to align along the $\mathrm{x}$ direction in the ANIS sample. The difference is important: as for xy-ISO samples there was no in-plane order, while in the ANIS samples molecular axes were mainly oriented along a given in-plane direction. The above procedure was then repeated piling up 16 layers: at each step the whole structure was very carefully relaxed. This effectively generated a $6.9 \mathrm{~nm}$-thick film of 752 TPD molecules, which resulted aligned parallel to the substrate (although with different in-plane arrangement, as discussed above). Eventually, any resulting sample sample was gently heated up (by a $10^{-4} \mathrm{~K} / \mathrm{fs}$ heating rate) and then equilibrated (400 ps $+100 \mathrm{ps}$ ) at the measurement temperature, obtaining a mass density in the range $1.080-1.085 \mathrm{~g} / \mathrm{cm}^{3}$, for the ANIS samples, and $1.069-1.079 \mathrm{~g} / \mathrm{cm}^{3}$ for the xy-ISO.

A different procedure was instead followed to generate a fully isotropic sample, which will be referred to as ISO. More specifically, the xy-ISO sample was used as starting configuration: the deposited film was heated up to $900 \mathrm{~K}$ during a 300 ps-long NVT run and then annealed for further 500 ps. The molecular orientation was carefully monitored on-the-fly to control the setup of a fully isotropic situation.

The TPD film was eventually cooled (at $10^{-4} \mathrm{~K} / \mathrm{fs}$ rate) and the equilibrated at the measurement temperature, resulting into a density in the range $1.059-1.065 \mathrm{~g} / \mathrm{cm}^{3}$.

Representative views of the structures are shown in Fig. 3. In short, the sequence (ISO)-(xy-ISO)-(ANIS) provides an increasing character of molecular anisotropy. 


\section{Results And DisCussion}

\subsection{Anisotropic thermal conductivity}

The calculated values of thermal conductivity resulted somewhat larger than those obtained experimentally. More specifically, at room temperature we get for the ISO sample $0.85 \mathrm{~W} \mathrm{~m}^{-1} \mathrm{~K}^{-1}$ to be compared with the experimental value $0.15 \mathrm{~W} \mathrm{~m}^{-1} \mathrm{~K}^{-1}$ [62]. This is very likely related to the adopted force-field, describing both intra- and intermolecular forces just approximately. MD simulations are nevertheless a powerful tool to provide trends and relative values. Therefore, in order to compare theoretical and experimental results, we opted for (i) normalizing any calculated thermal conductivity to the value obtained at $\mathrm{T}=320 \mathrm{~K}$ (corresponding to the simulated temperature closest to the actual experimental glass transition temperature) and (ii) averaging over the three main transport directions, i.e. setting $\left(\kappa_{\mathrm{xx}}+\kappa_{\mathrm{yy}}+\right.$ $\left.\kappa_{\mathrm{zz}}\right) / \kappa_{\mathrm{T}=320 \mathrm{~K}}=1$. The resulting picture is consistent with experimental evidence [62] as shown in Fig. 4 where the thermal conductivity of the investigated TPD films is reported for three the different directions. In particular, we remark that the ANIS sample is more efficient in carrying heat along the $\mathrm{x}$ direction than in any other direction and it also results more efficient than the ISO or xy-ISO samples.

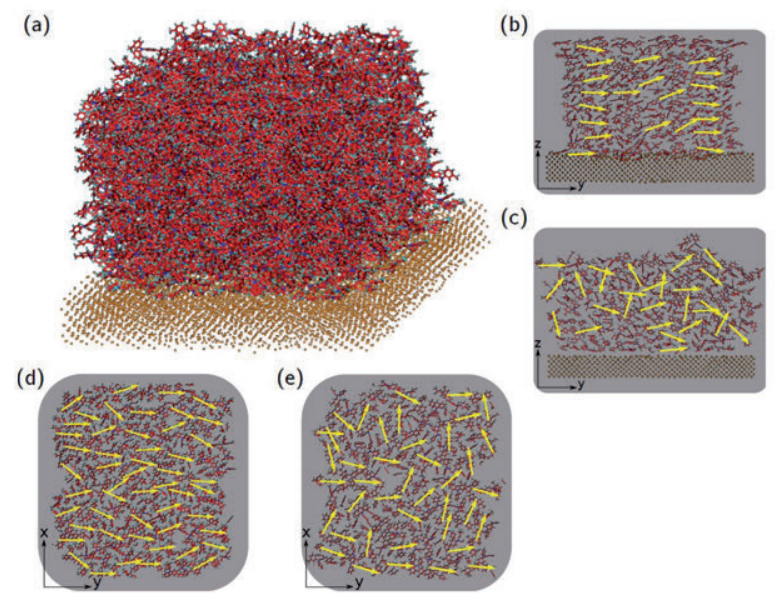

Fig. 3. Panel (a): perspective view of a TPD film deposited on a Si substrate.

Panels (b) and (c): side view of the xy-ISO and ISO sample, respectively.

Panels (d) and (e): top view of the ANIS and xy-ISO sample, respectively. Yellow arrows represent the orientation of the TPD molecular backbone. 
(a)

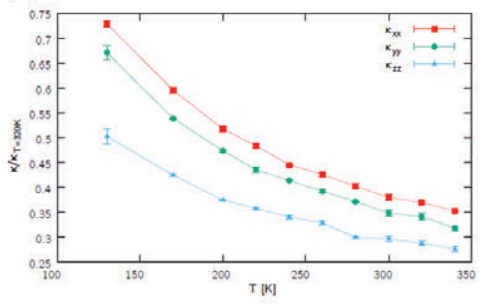

(b)

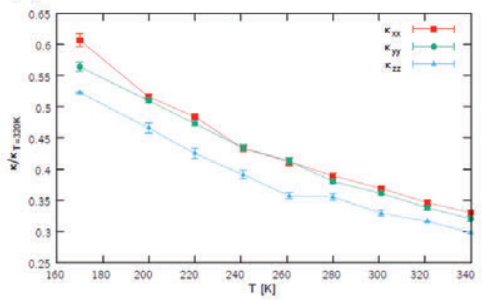

(c)

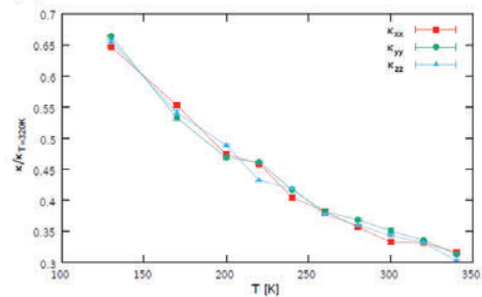

Fig. 4. Panel (a): thermal conductivity in the ANIS sample, normalized $\kappa_{\mathrm{T}=320 \mathrm{~K}}^{\mathrm{ANIS}}$ $a t=0.46 \mathrm{Wm}^{-1} \mathrm{~K}^{-1}$. Panel (b): hermal conductivity values in the xy-ISO sample, normalized at $\kappa_{\mathrm{T}=320 \mathrm{~K}}^{\mathrm{xy}-\mathrm{SOO}}=0.56 \mathrm{Wm}^{-1} \mathrm{~K}^{-1}$. Panel (c): thermal conductivity values in the ISO sample, normalized at $\kappa_{\mathrm{T}=320 \mathrm{~K}}^{\mathrm{ISO}}=0.85 \mathrm{Wm}^{-1} \mathrm{~K}^{-1}$.

Interesting enough, the ISO sample shows higher conductivity values along the $\mathrm{z}$ direction (i.e. along the through-plane direction): we argue that this is due to the enforced planar stacking parallel to the substrate occurring in both the xy-ISO and ANIS samples.

Finally, we investigated the relative difference in both the in-plane and the through-plane conductivities, respectively defined as

$$
\Delta \kappa_{\text {in-plane }}=\frac{\kappa_{\mathrm{xx}}-\kappa_{\mathrm{yy}}}{\kappa_{\mathrm{xx}}} \quad \Delta \kappa_{\text {through-plane }}=\frac{\kappa_{\|\|}-\kappa_{\perp}}{\kappa_{\|}}
$$

As shown in panel (a) of Fig. 5, thermal transport is largely affected by the molecular orientation, since $\Delta \kappa_{\text {in-plane }}$ is found to be a remarkable $\sim 9 \%$ for the ANIS sample, while it is very close to zero for the $\mathrm{xy}-$ ISO sample (as indeed expected for an isotropic configuration). In any case, the temperature effect is minor. This picture is fully confirmed by computing the through-plane relative difference as reported in panel (b) of Fig. 5. In this case temperature effects are more important, since by heating the ANIS sample we largely affect its preferential molecular alignment along the $\mathrm{x}$ direction. 
In summary, we argue that the substrate drives molecules to preferentially align their molecular backbone with in-plane orientation; furthermore, inherent anisotropy features of the TPD molecule are to a large extent preserved even at relatively high temperature.

(a)

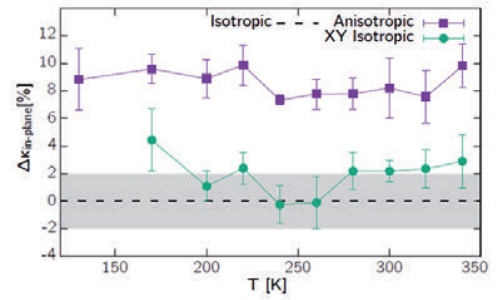

(b)

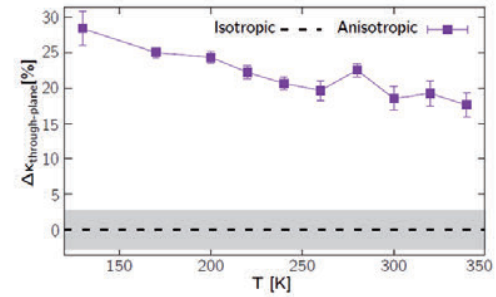

Fig. 5. Panel (a): relative variation of the in-plane thermal conductivity for the three systems investigated. Panel (b): relative variation of the through-plane thermal conductivity for the ANIS and the ISO samples.

\subsubsection{Molecular orientation}

More physical insight on transport features is provided by an indepth analysis of the molecular orientation. The spatial orientation of TPD molecules can be unambiguously specified by defining two vectors, namely: (i) the vector lying along the direction linking the nitrogen atoms of the molecule (hereafter labeled as NN-vector), shown in panel (a) of Fig. 6, and (ii) the vector identifying the direction normal to the plane containing the molecule (hereafter labeled as planar vector), shown in panel (b) of Fig. 6. This latter is defined by considering the vector product between the two vectors joining one nitrogen atom to two carbon atoms of the opposite aromatic ring of the TPD backbone.

(a)

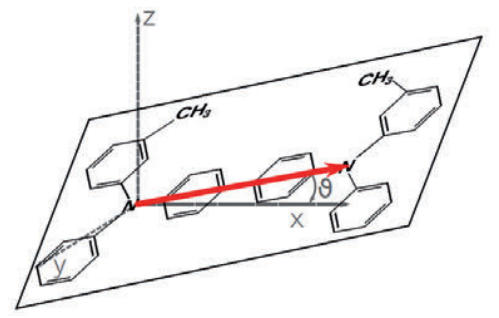

(b)

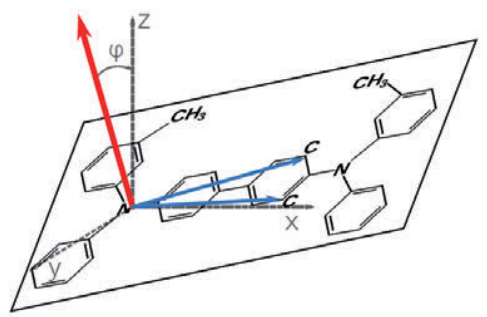

Fig. 6. Visualization of the NN-vector (panel a) and planar vector (panel b) for a single TDP molecule. Both vectors are shown in red color. 
The distribution of the angles formed by the NN-vector and the planar vector with the three cartesian axes (shown in Fig. 6 as dashed lines) provides information about the molecular orientations in each sample (we remind that the cartesian coordinates are defined as reported in Fig. 3). In Fig. 7 a thorough comparison between the ANIS and xy-ISO samples is performed by considering the orientation of the NN-vector (see panels (a), (b), and (c)); the ANIS and ISO samples are in turn compared by considering the orientation of the planar vector (see panels (d), (e), and (f)). All data are calculated at $\mathrm{T}=280 \mathrm{~K}$. The picture emerging from the MD simulations can be summarized as follows:

- Panel (a): in the ANIS sample the angular distribution of the NNvectors is peaked at $\cos \left(\theta_{\mathrm{x}}\right)= \pm 1$ which explains the higher efficiency of thermal transport along $\mathrm{x}$ direction. On the other hand, the xy-ISO sample shows a much flatter distribution due to its random orientation in the xy-plane.

- Panel (b): the ANIS sample shows a (broad) distribution around $\cos \left(\theta_{\mathrm{y}}\right)=0$, which is a fingerprint of a preferential normal orientation of the molecules with respect to the y direction. Once again, for the xy-ISO sample the distribution is much flatter and contains nonzero values for a wider range of $\cos \left(\theta_{\mathrm{y}}\right)$ values.

- Panel (c): both samples show a distribution peaked around $\cos \left(\theta_{z}\right)=0$, confirming the $\pi-\pi$ stacking along the $z$ direction: this reflects in a less efficient thermal transport.

- Panel (d): another interesting feature is that ANIS sample shows a distribution peaked around $\cos \left(\varphi_{\mathrm{x}}\right)=0$, suggesting that molecules are on average parallel to the xy-plane and aligned along $\mathrm{x}$. On the other hand, the ISO sample shows a broad and flatter distribution due to a random orientation of the molecular plane.

- Panel (e): the ANIS sample shows a flat distribution, corresponding to a randomly tilted orientation of the molecular plane with respect to y. This does not affect thermal transport since for such a system thermal conduction preferentially occurs along the $\mathrm{x}$ direction. The ISO sample has the same distribution shown in panel d.

- Panel (f): the distribution is peaked at $\cos \left(\varphi_{z}\right)= \pm 1$ for the ANIS sample, accounting for a preferential stacking arrangement along the $\mathrm{z}$ direction. The same distribution for the ISO sample, in turn, is almost flat as a consequence of the isotropy of the system. The small shoulders at $\cos \left(\varphi_{z}\right) \cong \pm 1$ are due to those molecules direct- 
ly attached to the substrate and to those molecules in the last plane of the TPD film, since they preserve an overall planar arrangement.

(a)
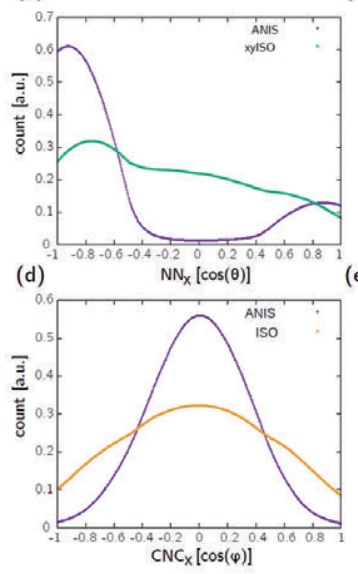

(b)
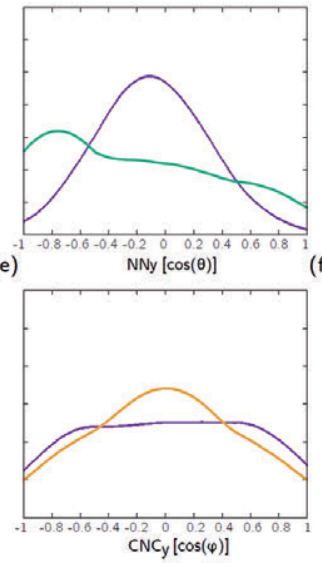

(c)
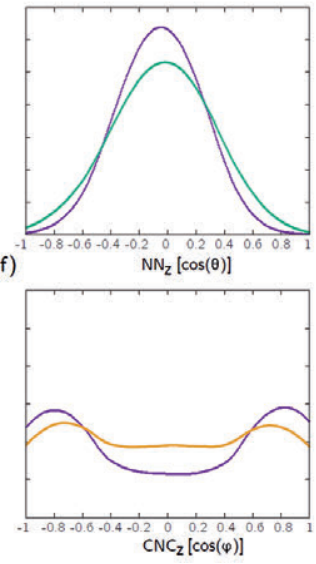

Fig. 7. Upper panels: angular distribution of the NN-vectors projected along the $x$ - (panel a), $y$ - (panel b), and z-direction (panel c) for the ANIS sample (purple lines) and the $x y$-ISO sample (green line). Lower panel: angular distribution of the planar-vectors projected along the $x$ - (panel d), $y$ - (panel e), and z-direction

(panel f) for the ANIS sample (purple lines) and the ISO sample (yellow line).

\subsection{A phenomenological model for TPD thermal conductance}

Anisotropic thermal transport suggests that a different heat flux is experienced by molecules oriented along the backbone direction or through $\pi-\pi$ stacking, which occurs in the normal-to-backbone direction.

In order to validate the above guess, two different quasi-1D structures were simulated, namely: (i) a linear bundle of TPD molecules aligned along the N-N axis and (ii) a line-up of TPD molecules aligned along the direction of $\pi-\pi$ bonding. Such configurations are shown in Fig. 8 and they will be hereafter referred to as backbone stacking or $\pi-\pi$ stacking, respectively. A structural unit containing $2 \times 2 \times 2$ TPD molecules is replicated along either backbone or $\pi-\pi$ stacking directions. The resulting structures were equilibrated with a low temperature NVT dynamics followed by an NPT relaxation for a total simula- 
tion time of 200 ps. The simulation cell was relaxed only along the direction of $1 \mathrm{D}$ alignment. Due to the TPD geometry, the $\pi-\pi$ configuration results more packed than the backbone chain and the same number of molecules produced a shorter $\pi-\pi$ stacking configuration. Both pseudo-1D samples were prepared with the same density, namely: $\rho_{\text {backbone }}=0.83 \mathrm{~g} / \mathrm{cm}^{3}$ and $\rho_{\pi-\pi \text { stacking }}=0.84 \mathrm{~g} / \mathrm{cm}^{3}$.

(a)

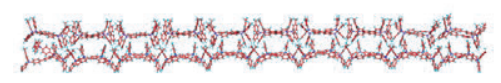

(b)

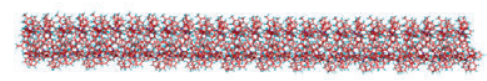

Fig. 8. Panel (a): structure realized organizing the TPD molecule along the backbone direction. Panel (b): structure realized piling up the molecule along the $\pi-\pi$ stacking direction.

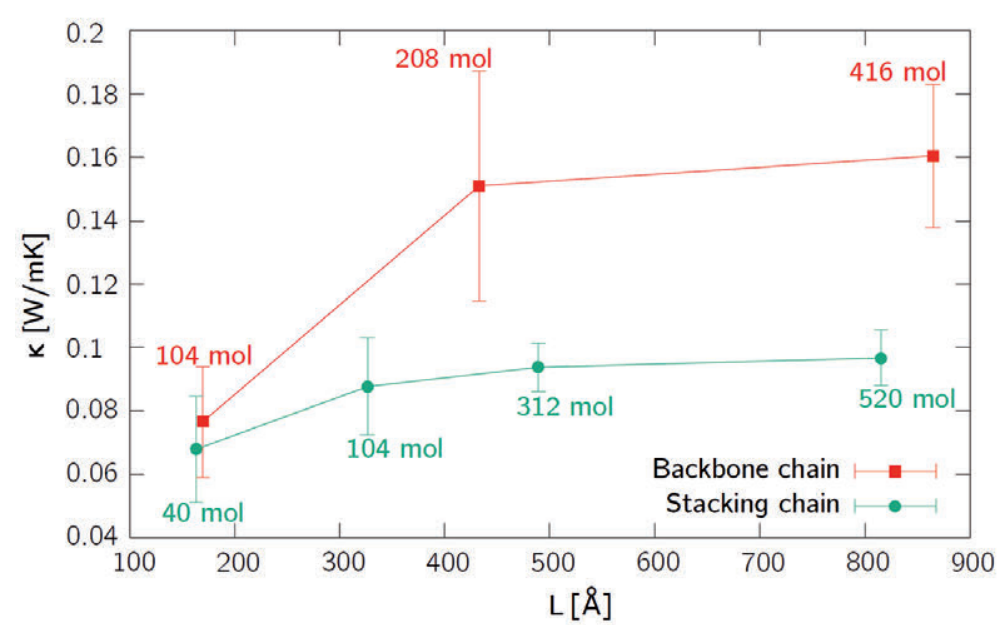

Fig. 9. Thermal conductivity as a function of the chain length $L$ for the quasi-1D structures.

\subsubsection{Stacking $v$ s backbone}

Fig. 9 reports the thermal conductivity $v$ s chain length $L$ for both stackings: a quite different upper limit is found for long enough systems, thus confirming that heat transport is favored along the backbone direction (70\% higher thermal conductivity). Moreover, a much larger number of TPD molecules is needed to reach the upper conductivity value for $\pi-\pi$ stacking, definitely confirming a less efficient transport mechanism along this direction. 


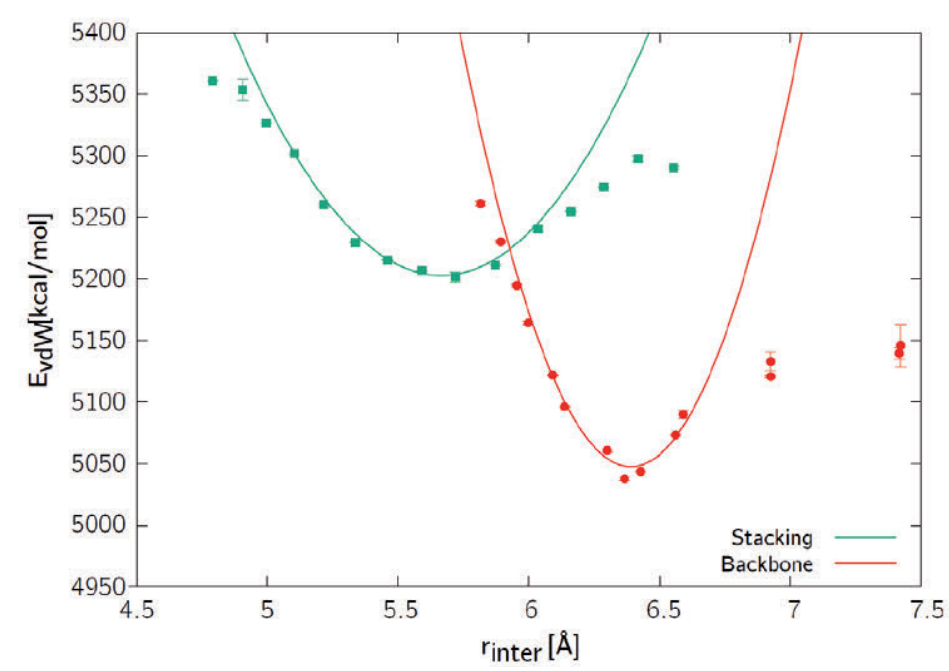

Fig. 10. Configurational energy $E_{v d W}$ due to van der Waals interactions only calculated as a function of the average intermolecular distance $r_{\text {inter. }}$.

\subsubsection{Intermolecular interactions}

In order to explain the results reported in the previous subsection, we evaluated the interaction strength between molecules for both stackings as function of the inter-molecular spacing.

The average intermolecular distance $r_{\text {inter }}$ was varied in the range $4.75 \AA \leq \mathrm{r}_{\text {inter }} \leq 7.5 \AA$ and the corresponding configurational energy has been computed as shown in Fig. 10. The resulting potential energy profile nearby the equilibrium distance is basically harmonic, while at smaller/higher distances the onset of anharmonic behavior is observed, as expected. A parabolic fitting near the minimum yields the effective force constant $\mathrm{K}$ characterizing the intermolecular coupling within a simple spring-and-ball picture according to

$$
\mathrm{E}(\mathrm{r})=\mathrm{K}\left(\mathrm{r}-\mathrm{r}_{0}\right)^{2}+\mathrm{E}_{0}
$$

where $\mathrm{E}_{0}$ is the energy minimum located at $\mathrm{r}_{0}$. We calculated $\mathrm{K}_{\text {backbone }}=$ $824.7 \mathrm{kcal} / \mathrm{mol} \AA^{2}, \mathrm{r}_{\text {backbone }}=6.39 \AA$ and $\mathrm{E}_{0, \text { backbone }}=5047.2 \mathrm{kcal} / \mathrm{mol}$, for the backbone configuration and $\mathrm{K}_{\text {stacking }}=312.7 \mathrm{kcal} / \mathrm{mol} \AA^{2}, \mathrm{r}_{\text {stacking }}=$ $5.67 \AA$ and $\mathrm{E}_{0 \text {,stacking }}=5202.8 \mathrm{kcal} / \mathrm{mol}$ for the stacking configuration.

Consistently with the adopted picture, it can be argued that a stiffer effective spring value translates into a more efficient thermal conduction, 
according to the following twofold heuristic argument. In general, thermal conductivity is proportional to the group velocity of heat carriers: since a larger force constant causes a steeper vibrational branch, this reflects into a higher group velocity. On the other hand, heat current basically represents the energy transferred by a flux of carriers corresponding to atomic vibrations: the higher the force constant, the higher the vibrational energy, the higher the energy of such heat carriers. The thermal anisotropy reported here qualitatively agrees with observations in aligned polymers systems where an increase in the strength of intermolecular forces leads to an enhancement of the thermal conductivity [53]. The in-plane and throughplane conductance can be estimated considering that the molecules and $\mathrm{vdW}$ interactions form a thermal resistive network, as shown in Fig. 11.
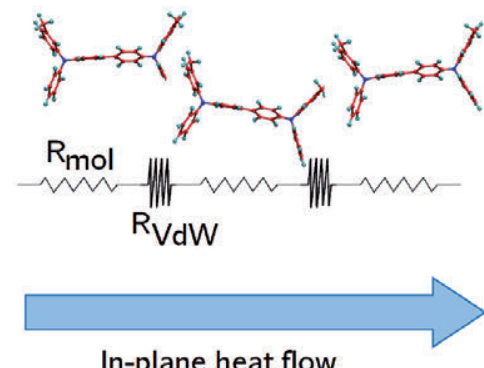

In-plane heat flow

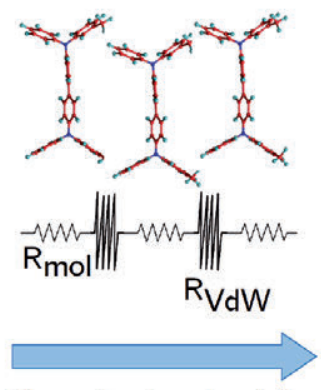

Through-plane heat flow

Fig. 11. Schematics of thermal resistance network in the in-plane direction (left) and the through-plane direction (right). $R_{v d W}$ and $R_{\text {mol }}$ account for the strength of the inter-molecular and intra-molecular interactions, respectively.

The interface thermal resistance (ITR), a measure of the effective thermal coupling between neighboring molecules joined by weak vdW interactions, is assumed to dominate thermal transport if both molecules and vdW interactions can be seen as forming a thermal resistive network. The stronger molecular interaction effectively corresponds to a lower ITR (higher thermal interface conductance), while the intermolecular $\pi-\pi$ stacking entails higher thermal resistance between molecules. Within such a network model, the total thermal resistance can be written as the sum of the two series resistances $R_{T}=R_{T, m o l}+R_{T, v d W}=$ $N_{\text {mol }} R_{\text {mol }}+N_{v d W} R_{v d W}$, where $N_{\text {mol }}$ and $N_{v d W}$ are the number of molecular and vdW units, respectively, while $R_{m o l}$ and $R_{v d W}$ are the resistances associated to each unit, respectively. 
yields to

Writing the above $R_{T}$ in terms of the individual conductivities

$\mathrm{R}_{\mathrm{T}}=\mathrm{N}_{\mathrm{mol}} \frac{\mathrm{L}_{\mathrm{mol}}}{\kappa_{\mathrm{mol}}}+\mathrm{N}_{\mathrm{vdW}} \frac{\mathrm{L}_{\mathrm{vdW}}}{\kappa_{\mathrm{vdW}}}$

with $\mathrm{L}_{\mathrm{mol}}$ and $\mathrm{L}_{\mathrm{vdw}}$ being the lengths of the single units. Therefore, we can write the total conductance (along a specific direction) as

$\mathrm{G}_{\mathrm{T}}=\frac{\kappa}{\mathrm{L}}=\frac{1}{\mathrm{R}_{\mathrm{T}}}=\frac{\kappa_{\mathrm{mol}} \kappa_{\mathrm{vdW}}}{\kappa_{\mathrm{vdW}} \mathrm{N}_{\mathrm{mol}} \mathrm{L}_{\mathrm{mol}}+\kappa_{\mathrm{mol}} \mathrm{N}_{\mathrm{vdW}} \mathrm{L}_{\mathrm{vdW}}}$

where $\mathrm{L}$ is the total sample length and $\kappa$ is the computed thermal conductivity (along a specific direction). Assuming that the conductance is dominated by an ITR due to vdW interactions, we guess $\kappa_{\mathrm{mol}}>>\kappa_{\mathrm{vdW}}$ leading to a simplified expression

$\mathrm{G}_{\mathrm{T}} \cong \frac{\kappa_{\mathrm{mol}} \kappa_{\mathrm{vdW}}}{\kappa_{\mathrm{mol}} N_{\mathrm{vdW}} L_{\mathrm{vdW}}}=\frac{\mathrm{G}_{\mathrm{vdW}}}{\mathrm{N}_{\mathrm{vdW}}}$

Combining Eqs. (10) and (11), an expression of the thermal interface conductance for the interatomic interactions is obtained

$$
\mathrm{G}_{\mathrm{vdW}}=\kappa \frac{N_{\mathrm{vdW}}}{\mathrm{L}}=\frac{\kappa}{\mathrm{L}_{\mathrm{mol}}+\mathrm{L}_{\mathrm{vdW}}}
$$

Where $G_{v d W}$ stands for the conductance of an individual unit (the interface thermal conductance due to $\mathrm{vdW}$ interactions).

(a)

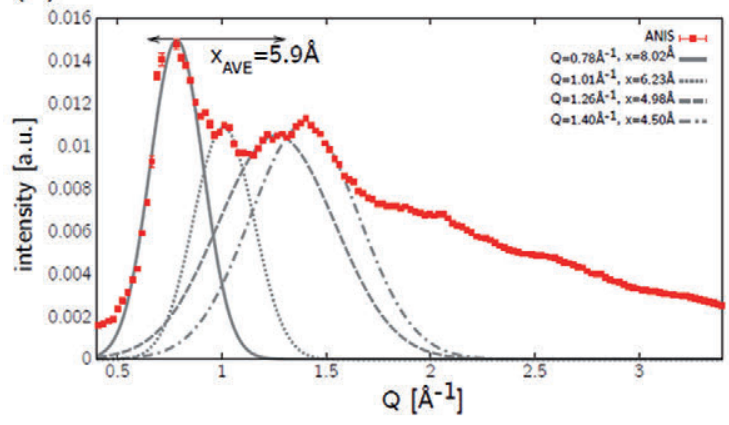

(b)

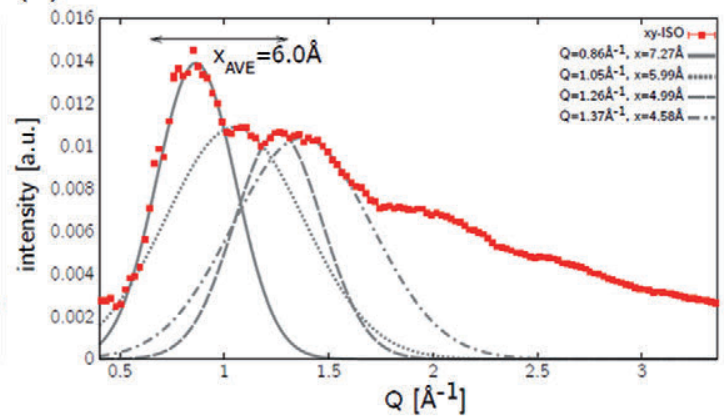

Fig. 12. Panel (a): simulated XRD diffraction pattern of the ANIS sample.

Panel (b): simulated XRD diffraction pattern of the xy-ISO sample. The major contribution is located around $1.4 A^{-1}$ and it is associated with the planar stacking of the molecules since it corresponds to the typical distance in the $\pi-\pi$ stacking configurations. The other peaks at lower angles are relative to the different orientation of the molecule in-plane and an amplitude-weighted average produces a distance around $6 \AA$. Red squared symbols correspond to the calculated profile, while black lines provide a Gaussian fit for the different peaks. 
In order to use eq. (12) we need to evaluate the average distance between molecules, i.e. the $\mathrm{L}=\mathrm{L}_{\mathrm{mol}}+\mathrm{L}_{\mathrm{vdW}}$ term, for both the in-plane and the through-plane directions. This quantity has been evaluated from the simulated XRD profiles reported in Fig. 12 which, in turn, have been calculated following the procedure described in Ref. [63] for both the ANIS and xy-ISO samples at T $=300 \mathrm{~K}$ with an incident wavelength $\lambda=1.541 \AA$ as used in similar GIWAXS studies [64, 65]. The $\mathrm{XRD}$ patterns were calculated by considering only the nitrogen atoms belonging to different TPD molecules. This choice allowed to unveil the inter-diffraction chain structural features discarding the intramolecular peaks.

The simulated average distances are, respectively, $4.50 \AA$ ( $4.58 \AA)$ and $5.9 \AA(6.0 \AA)$ in the through-plane and in-plane, respectively, for the ANIS (xy-ISO) sample. Experimentally [67] the distance between molecules in the through-plane direction is well defined by the lowangle XRD peak located at $\mathrm{Q} \sim 1.4 \AA^{-1}$ providing a $4.5 \AA$ value, indeed very close to our calculated one. The in-plane counterpart is more difficult to evaluate experimentally, since several low-angle peaks or shoulders appear at $1.2,1.0$ and $0.75 \AA^{-1}$. A rough average provides a mean distance value of about $6 \AA$, once again close to our results. The agreement between simulated and experimental data support the suitability of the simulated structure.

We can now finalize the thermal conductance calculation. According to Fourier law, the heat flux $\mathrm{J}$ is proportional to the temperature difference $\Delta \mathrm{T}$ through $\mathrm{J}=\mathrm{G} \Delta \mathrm{T}$, where $\mathrm{G}$ is the thermal conductance. Considering the in-plane and through-plane values of the thermal conductivity obtained for the experimental samples [62] at $\mathrm{T}=300 \mathrm{~K}$ (i.e. $\kappa_{\|}=$ $0.175 \mathrm{~W} \mathrm{~m}^{-1} \mathrm{~K}^{-1}$ and $\kappa_{\perp}=0.110 \mathrm{~W} \mathrm{~m}^{-1} \mathrm{~K}^{-1}$ ), we get the following results: $\mathrm{G}_{\mathrm{vdW}, \|} \sim 292 \mathrm{MW} \mathrm{m}^{-2} \mathrm{~K}^{-1}$ and $\mathrm{G}_{\mathrm{vdW}, \perp} \sim 240 \mathrm{MW} \mathrm{m}{ }^{-2} \mathrm{~K}^{-1}$. In order to establish whether the present estimation is meaningful, we remind for a metal/dielectric interface it is typically reported approximately a conductance as high as $1 \mathrm{GW} \mathrm{m}$ m $^{-1}$; on the other hand, the calculated thermal interface conductance between different crystallographic orientations in crystalline dinaphtho[2,3-b:2,3-f]thieno[3,2-b]thiophene (DNTT) ranges between 150-300 $\mathrm{MW} \mathrm{m}^{-2} \mathrm{~K}^{-1}$ [67] while for an interface between myoglobin proteins it amounts to $301 \mathrm{MWm}^{-2} \mathrm{~K}^{-1}$ at $320 \mathrm{~K}$ [68]. The present data for TPD-base systems lie between those found for organic-organic systems such as the copper phthalocyanine $(\mathrm{CuPc})$-fullerene $\left(\mathrm{C}_{60}\right)$ interface, $\left(400 \mathrm{MW} \mathrm{m}{ }^{-2} \mathrm{~K}^{-1}\right)$ and those found for organic/inorganic interfaces 
such as pentacene/metal $\left(10 \mathrm{MW} \mathrm{m}{ }^{-2} \mathrm{~K}^{-1}\right)$ [69] or CuPc-Au $\left(20 \mathrm{MW} \mathrm{m}{ }^{-2}\right.$ $\mathrm{K}^{-1}$ ) that is purely a vdW-like interaction [70].

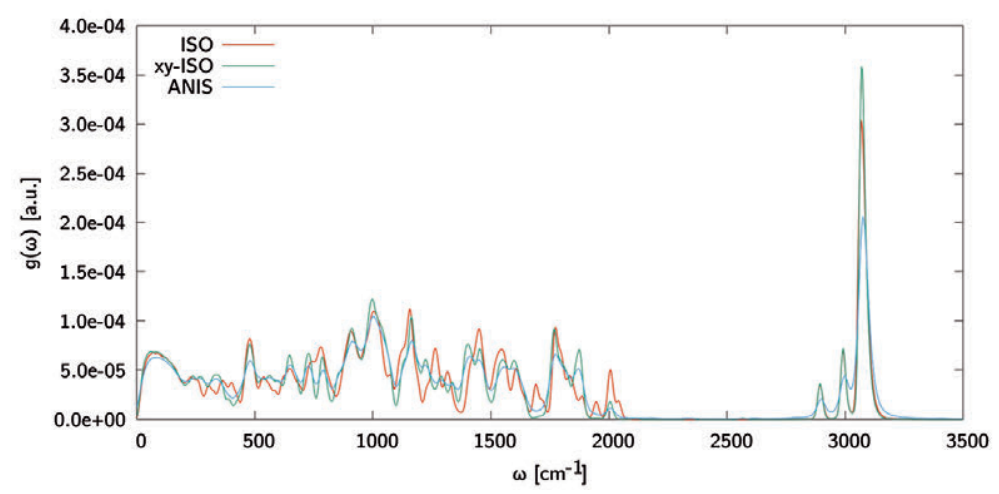

Fig. 13. Vibrational density of state calculated for the three samples investigated.

\subsection{Heat capacity of TPD}

In order to complete the investigation on the thermal properties of TPD glassy films, we calculated their constant-volume heat capacity $\mathrm{C}_{\mathrm{v}}^{\mathrm{QHA}}(\mathrm{T})$ within the quasi-harmonic approximation [71] as

$$
\mathrm{C}_{v}^{\mathrm{QHA}}(\mathrm{T})=3 \mathrm{Nk}_{\mathrm{B}} \int_{0}^{\infty} \mathrm{d} \omega \mathrm{g}(\omega) \mathrm{F}\left(\frac{\hbar \omega}{\mathrm{k}_{\mathrm{B}} \mathrm{T}}\right)
$$

where the vibrational density of states (vDOS) $g(\omega)$ is integrated considering the Bose-Einstein weighing factor

$$
\mathrm{F}\left(\frac{\hbar \omega}{\mathrm{k}_{\mathrm{B}} \mathrm{T}}\right)=\left(\frac{\hbar \omega}{\mathrm{k}_{\mathrm{B}} \mathrm{T}}\right)^{2} \exp \left(\frac{\hbar \omega}{\mathrm{k}_{\mathrm{B}} \mathrm{T}}\right) /\left[\exp \left(\frac{\hbar \omega}{\mathrm{k}_{\mathrm{B}} \mathrm{T}}\right)-1\right]^{2},
$$

where $\omega$ are the frequencies of the $3 \mathrm{~N}$ vibrational modes in the TPD film.

In order to determine the vibrational spectra, we followed the procedure described in Ref. [72] by building and diagonalizing the corresponding dynamical matrix

$$
\mathrm{D}_{\mathrm{i} \alpha, i \beta}=-\frac{1}{\sqrt{\mathrm{m}_{\mathrm{i}} \mathrm{m}_{\mathrm{j}}}} \frac{\partial \mathrm{F}_{\mathrm{i} \alpha}}{\partial \mathrm{r}_{\mathrm{j} \beta}}
$$

where we considered the gradients of the force $F_{i \alpha}$ acting on the i-th atom with mass $\mathrm{m}_{\mathrm{i}}$ along the $\alpha$-direction caused by an infinitesimal displacement of the $j$-th atom with mass $m_{j}$ along the $\beta$-direction. Here $i, j=1,2, \ldots, N$ where $\mathrm{N}$ counts for the total number of atoms in the simulation cell and $\alpha$ 
and $\beta$ indicate the Cartesian coordinates. MD simulations provide direct computation of $F_{i}$ and the first order derivatives have been numerically computed by finite difference with an atomic displacement as small as $5 \times 10^{-4} \AA$. From the diagonalization of the dynamical matrix, eigenvectors $e_{s}$ and eigenvalues $\omega_{s}^{2}$ are obtained, where $s=1, \ldots, 3 \mathrm{~N}$ counts eigenmodes. We used the SLEPc library for matrix diagonalization [73-76]. This procedure allowed us to calculate the vDOS of the aforementioned three samples ISO, xy-ISO, and ANIS as reported in Fig. 13. The density of vibrational states does not change appreciably for the two anisotropic TPD systems: we argue that the vibrational properties are mostly influenced by the planar stacking of the molecules with respect to the substrate, as already pointed out by thermal conductivity calculations. On the other hand, the vDOS shows remarkable variations when comparing isotropic and anisotropic samples: the differences mostly occur in the region 1000-2000 $\mathrm{cm}^{-1}$, which characterize the C-C and C-N stretching modes.

The calculated $\mathrm{C}_{\mathrm{v}}{ }^{\mathrm{OHA}}(\mathrm{T})$ are reported in Fig. 14 and compared with the available high- temperature experimental data [62]: the agreement is just very good, thus establishing the reliability of the present theoretical framework.

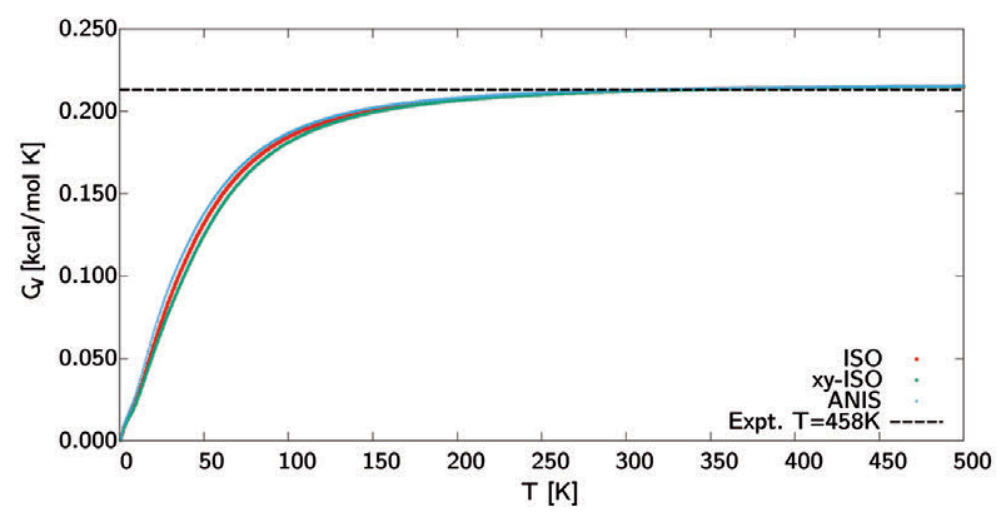

Fig. 14. Calculated heat capacity (quasi-harmonic approximation) of three TPD glassy samples.

It is interesting to focus on the low temperature regime, which is shown in Fig. 15. While for a 3-dimensional assembly of harmonic oscillators it is predicted $\mathrm{C}_{\mathrm{v}}{ }^{\mathrm{OHA}}(\mathrm{T}) \sim \mathrm{T}^{3}$ (a behavior typical of inorganic crystalline materials successfully established by Debye theory), we observe that for any TPD film here investigated a rather different - and more complex trend is observed, regardless the degree of isotropicity. In particular: 
- In the very low temperature regime (i.e. below $2 \mathrm{~K}$ ) we observe that the heat capacity increases linearly with temperature: this feature has been found as well in many ordinary glassy or amorphous materials [77-80] and it is usually interpreted as tunneling of atoms (or groups of atoms) between two (or more) possible equilibrium positions characterized by similar energy (two levels system model). An asymmetric double-well potential is in general used for describing local rearrangements of atoms.

- In the upper $10 \mathrm{~K}-15 \mathrm{~K}$ temperature range the temperature dependence abruptly change. However, while ordinary glassy materials in this range recover the standard $\sim \mathrm{T}^{3}$ dependence, our calculations unambiguously show that this is not the case for the investigated TPD glasses. More specifically, by fitting the calculated values for the ISO sample the range $4 \mathrm{~K}<\mathrm{T}<10 \mathrm{~K}$, we clearly get a heat capacity varying as $\mathrm{C}_{\mathrm{v}}^{\mathrm{QHA}}(\mathrm{T}) \sim \mathrm{T}^{3 / 2}$. Similar results are obtained for the anisotropic samples. We attribute this remarkable difference with respect to the standard behavior to the very nature of the organic glasses: we argue that the molecular nature of the TPD system does not match the concept of an ensemble of independent (harmonic) atomic oscillators underlying the Debye theory for the heat capacity of a crystalline solid. In fact, organic molecules are rather complex systems which, besides the stretching of the intramolecular bonds due to the oscillations of the atoms around their equilibrium positions, are characterized by quite different degrees of freedom, including bond-bending and librations of several different bond-angles.

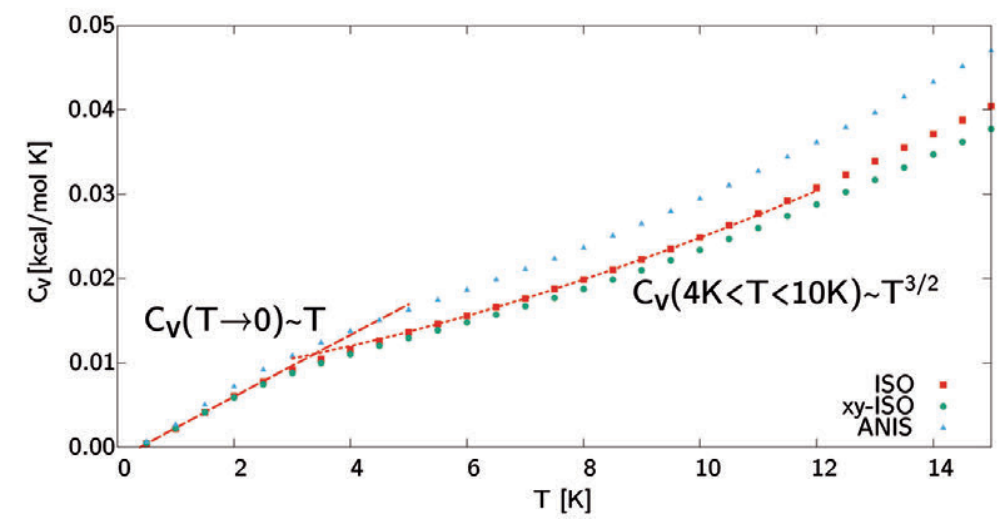

Fig. 15. Calculated $C_{v}{ }^{\mathrm{OHA}}(\mathrm{T})$ at low temperature (dots).

The dashed and dotted line represents the fitting behavior for the ISO sample, respectively below $2 \mathrm{~K}$ and in the rage $4 \mathrm{~K}<T<10 \mathrm{~K}$. 
We believe that the here predicted anomalous behavior of the heat capacity in the low temperature regimes could play an important role in the ongoing search for possible boson peak effects in organic glasses. As a matter of fact, in order to assess the possible existence of such a peak, one should compare measured (or computed) data to a reference theoretical model which, according to present results, should not be the typical Debye-like $\sim \mathrm{T}^{3}$ behavior.

Further investigations are, therefore, required in order to provide a detailed explanation of the observed trends: in particular, a critical readdressing of the theoretical framework for the evaluation of the heat capacity could explain the different T-dependence with respect to inorganic amorphous or crystalline materials, possibly providing a universal low-temperature behavior for organic glasses.

\section{Conclusions}

In this work we performed atomistic simulations to investigate thermal properties of TPD-based organic semiconductor glassy systems, exhibiting interesting structural features related to the strongly anisotropic morphology of the constitutive molecule.

In particular, we realized three different samples, characterized by different degrees of anisotropy, and we calculated thermal conductivity in order to highlight possible thermal anisotropy. The results have been correlated to the efficiency in transferring heat along different directions: in particular, we found that the intermolecular conductance characterizing the connections along the N-N axis of the molecule (i.e. those identified by the $\sigma-\sigma$ bond) are much higher than that occurring along the direction of the $\pi-\pi$ stacking. We attributed this difference to an increased intensity in the intermolecular interactions.

In addition, we provided the first theoretical evaluation of the heat capacity $\mathrm{C}_{\mathrm{v}}$ of a TPD-based glassy system in the quasi-harmonic approximation framework. We provide the first evidence that the lowtemperature heat capacity strongly deviates from the typical $\mathrm{C}_{\mathrm{v}} \sim \mathrm{T}^{3}$ behavior predicted for inorganic systems, although the linear trend for very low temperatures is still observed. 


\section{REFERENCES}

[1] D. Turnbull. "Under what conditions can a glass be formed?" Contemporary Physics 10.5 (1969), 473-488.

[2] C.A. Angell. "Structural instability and relaxation in liquid and glassy phases near the fragile liquid limit." J. Non-Cryst. Solids 102.1 (1988). Proceedings of the Ninth University Conference on Glass Science, 205-221.

[3] C.T. Moynihan, P.B. Macedo, C.J. Montrose, P.K. Gupta, M.A. DeBolt, J.F. Dill, B.E. Dom, P.W. Drake, and A.J. Easteal et al. "Thermodynamic and transport properties of liquids near the glass transition temperature. Structural relaxation in vitreous materials." In: The Glass Transition and the Nature of the Glassy State. Ed. by M. Goldstein and R. Simha. Vol. 279. New York Academy of Sciences, 1976.

[4] R. Brüning and K. Samwer. "Glass transition on long time scales." Phys. Rev. B 46.18 (1992), 11318-11322.

[5] P.G. Debenedetti and F.H. Stillinger. "Supercooled liquids and the glass transition." Nature 410 (2001), 259.

[6] F.H. Stillinger and T.A. Weber. "Computer simulation of local order in condensed phases of silicon.” Phys. Rev. B 31.8 (1985), 5262-5271.

[7] M.D. Ediger, C.A. Angell, and S.R. Nagel. "Supercooled Liquids and Glasses." J. Phys. Chem. 100.31 (1996), 13200-13212.

[8] W. Kauzmann. "The Nature of the Glassy State and the Behavior of Liquids at Low Temperatures." Chemical Reviews 43.2 (1948), 219-256.

[9] F. Simon. "er den Zustand der unterkhlten Flüssigkeiten und Gläser." Zeitschrift für anorganische und allgemeine Chemie 203.1 (1931), 219-227.

[10] P.G. Wolynes. "Aperioidic crystals: Biology, Chemistry and Physics in a fugue with stretto.” AIP Conference Proceedings 180.1 (1988), 39-65.

[11] P.G. Wolynes. "Entropy Crises in Glasses and Random Heteropolymers." J Res. Natl. Inst. Stand. Technol. 102.2 (1997), 187-194.

[12] C.A. Angell. "Formation of Glasses from Liquids and Biopolymers." Science 267.5206 (1995), 1924-1935.

[13] B. Geffroy, P. le Roy, and C. Prat. "Organic light-emitting diode (OLED) technology: materials, devices and display technologies.” Polymer International 55.6 (2006), 572-582.

[14] S. Reineke, M. Thomschke, B. Lüssem, and K. Leo. "White organic light-emitting diodes: status and perspective." Rev. Mod. Phys. 85.3 (2013), 1245-1293.

[15] J.R. Sheats, H. Antoniadis, M. Hueschen, W. Leonard, J. Miller, R. Moon, D. Roitman, and A. Stocking. "Organic Electroluminescent Devices." Science 273.5277 (1996), 884-888.

[16] X. Zhou, J. He, L.S. Liao, M. Lu, X.M. Ding, X.Y. Hou, X.M. Zhang, X.Q. He, and S.T. Lee. "Real-Time Observation of Temperature Rise and Thermal Breakdown Processes in Organic LEDs Using an IR Imaging and Analysis System.” Adv. Mater. 12.4 (2000), 265-269.

[17] S. Chung, J.H. Lee, J. Jeong, J.J. Kim, and Y. Hong. "Substrate thermal conductivity effect on heat dissipation and lifetime improvement of organic light-emitting diodes.” Appl. Phys. Lett. 94.25 (2009), 253302. 
[18] X. Wang, K.D. Parrish, J.A. Malen, and P.K.L. Chan. "Modifying the thermal conductivity of small molecule organic semiconductor thin films with metal nanoparticles." Sci. Rep. 5 (2015), 16095.

[19] O. Bubnova, Z.U. Khan, A. Malti, S. Braun, M. Fahlman, M. Berggren, and X. Crispin. "Optimization of the thermoelectric figure of merit in the conducting polymer poly(3,4-ethylenedioxythiophene).” Nat. Mater. 10 (2011), 429 EP.

[20] Q. Zhang, Y. Sun, W. Xu, and D. Zhu. "Organic Thermoelectric Materials: Emerging Green Energy Materials Converting Heat to Electricity Directly and Efficiently.” Adv. Mater. 26.40 (2014), 6829-6851.

[21] T.A. Lin et al. "Sky-Blue Organic Light Emitting Diode with 37Quantum Efficiency Using Thermally Activated Delayed Fluorescence from Spiroacridine-Triazine Hybrid.” Adv. Mater. 28.32 (2016), 6976-6983.

[22] D. Mendels and N. Tessler. "Drift and Diffusion in Disordered Organic Semiconductors: The Role of Charge Density and Charge Energy Transport." J. Phys. Chem. C 117.7 (2013), 3287-3293.

[23] K.P. Pernstich, B. Rössner, and B. Batlogg. "Field-effect-modulated Seebeck coefficient in organic semiconductors.” Nat. Mater. 7 (2008), 321.

[24] S.F. Swallen, K.L. Kearns, M.K. Mapes, Y.S. Kim, R.J. McMahon, M.D. Ediger, T. Wu, L. Yu, and S. Satija. "Organic Glasses with Exceptional Thermodynamic and Kinetic Stability." Science 315.5810 (2007), 353-356.

[25] S.S. Dalal, D.M. Walters, I. Lyubimov, J.J. de Pablo, and M.D. Ediger. "Tunable molecular orientation and elevated thermal stability of vapor-deposited organic semiconductors." Proc. Natl. Acad. Sci. U.S.A. 112.14 (2015), 4227-4232.

[26] D.M. Walters, R. Richert, and M.D. Ediger. "Thermal stability of vapor-deposited stable glasses of an organic semiconductor.” J. Chem. Phys. 142.13 (2015), 134504.

[27] K.L. Kearns, S.F. Swallen, M.D. Ediger, T. Wu, and L. Yu. "Influence of substrate temperature on the stability of glasses prepared by vapor deposition." J. Chem. Phys. 127.15 (2007), 154702.

[28] E. Leon-Gutierrez, A. Sepulveda, G. Garcia, M.T. Clavaguera-Mora, and J. Rodriguez-Viejo. "Stability of thin film glasses of toluene and ethylbenzene formed by vapor deposition: an in situ nanocalorimetric study." Phys. Chem. Chem. Phys. 12.44 (2010), 14693-14698.

[29] M. Ahrenberg, Y.Z. Chua, K.R. Whitaker, H. Huth, M.D. Ediger, and C. Schick. "In situ investigation of avapor-deposited glasses of toluene and ethylbenzene via alternating current chip-nanocalorimetry.” J. Chem. Phys. 138.2 (2013), 024501.

[30] S.L.L.M. Ramos, M. Oguni, K. Ishii, and H. Nakayama. "Character of Devitrification, Viewed from Enthalpic Paths, of the Vapor-Deposited Ethylbenzene Glasses.” J. Phys. Chem. B 115.49 (2011), 14327-14332.

[31] C. Rodríguez-Tinoco, M. Gonzalez-Silveira, J. Ràfols-Ribé, G. Garcia, and J. Rodriguez-Viejo. "Highly stable glasses of celecoxib: Influence on thermo-kinetic properties, microstructure and response towards crystal growth." J. NonCryst. Solids 407. Supplement C (2015), 256-261.

[32] K.R. Whitaker, M. Tylinski, M. Ahrenberg, C. Schick, and M.D. Ediger. "Kinetic stability and heat capacity of vapor-deposited glasses of o-terphenyl." J. Chem. Phys. 143.8 (2015), 084511. 
[33] K.L. Kearns, S.F. Swallen, M.D. Ediger, Y. Sun, and L. Yu. "Calorimetric Evidence for Two Distinct Molecular Packing Arrangements in Stable Glasses of Indomethacin.” J. Phys. Chem. B 113.6 (2009), 1579-1586.

[34] S.S. Dalal, Z. Fakhraai, and M.D. Ediger. "High-Throughput Ellipsometric Characterization of Vapor-Deposited Indomethacin Glasses.” J. Phys. Chem. B 117.49 (2013), 15415-15425.

[35] K. Ishii, H. Nakayama, S. Hirabayashi, and R. Moriyama. "Anomalously high-density glass of ethylbenzene prepared by vapor deposition at temperatures close to the glass transition temperature." Chem. Phys. Lett. 459.1 (2008), 109-112.

[36] S.S. Dalal, A. Sepúlveda, G.K. Pribil, Z. Fakhraai, and M. D. Ediger. "Density and birefringence of a highly stable $\alpha, \alpha, \beta$-trisnaphthylbenzene glass." J. Chem. Phys. 136.20 (2012), 204501.

[37] Y.Z. Chua, M. Ahrenberg, M. Tylinski, M.D. Ediger, and C. Schick. "How much time is needed to form a kinetically stable glass? AC calorimetric study of vapor deposited glasses of ethylcyclohexane.” J. Chem. Phys. 142.5 (2015), 054506.

[38] K.J. Dawson, K.L. Kearns, M.D. Ediger, M.J. Sacchetti, and G.D. Zografi. "Highly Stable Indomethacin Glasses Resist Uptake of Water Vapor." J. Phys. Chem. B 113.8 (2009), 2422-2427.

[39] K.L. Kearns, T. Still, G. Fytas, and M.D. Ediger. "High-Modulus Organic Glasses Prepared by Physical Vapor Deposition.” Adv. Mater. 22.1 (2010), 39-42.

[40] J. Ràfols Ribé. "Organic Vapour-Deposited Stable Glasses: From Fundamental Thermal Properties to High-Performance Organic Light Emitting Diodes.” $\mathrm{PhD}$ thesis. Universitat Autònoma de Barcelona, 2017.

[41] T.D. Schmidt, T. Lampe, M.R.D. Sylvinson, P.I. Djurovich, M.E. Thompson, and W. Brütting. "Emitter Orientation as a Key Parameter in Organic LightEmitting Diodes.” Phys. Rev. Appl. 8.3 (2017), 037001.

[42] H.W. Lin, C.L. Lin, H.H. Chang, Y.T. Lin, C.C. Wu, Y.M. Chen, R.T. Chen, Y. Y. Chien, and K.T. Wong. "Anisotropic optical properties and molecular orientation in vacuum-deposited ter(9,9-diarylfluorene)s thin films using spectroscopic ellipsometry.” J. Appl. Phys. 95.3 (2004), 881-886.

[43] D. Yokoyama, A. Sakaguchi, M. Suzuki, and C. Adachi. "Enhancement of electron transport by horizontal molecular orientation of oxadiazole planar molecules in organic amorphous films.” Appl. Phys. Lett. 95.24 (2009), 243303.

[44] D. Yokoyama. "Molecular orientation in small-molecule organic light-emitting diodes.” J. Mater. Chem. 21.48 (2011), 19187-19202.

[45] S. Fratini, S. Ciuchi, D. Mayou, G. Trambly de Laissardière, and A. Troisi. "A map of high-mobility molecular semiconductors.” Nat. Mater. 16 (2017), 998.

[46] N. Kim, B. Domercq, S. Yoo, A. Christensen, B. Kippelen, and S. Graham. "Thermal transport properties of thin films of small molecule organic semiconductors." Appl. Phys. Lett. 87.24 (2005), 241908.

[47] N. Lu, L. Li, N. Gao, and M. Liu. "A unified description of thermal transport performance in disordered organic semiconductors." Org. Electron. 41. Supplement C (2017), 294-300.

[48] C.J. Glassbrenner and G.A. Slack. "Thermal Conductivity of Silicon and Germanium from $3^{\circ} \mathrm{K}$ to the Melting Point.” Phys. Rev. 134.4A (1964), A1058A1069. 
[49] D.G. Cahill, M. Katiyar, and J.R. Abelson. "Thermal conductivity of a-Si: H thin films." Phys. Rev. B 50.9 (1994), 6077-6081.

[50] D.G. Cahill and R.O. Pohl. "Heat flow and lattice vibrations in glasses." Solid State Commun. 70.10 (1989), 927-930.

[51] H. Zhang and J.W. Brill. "Interlayer thermal conductivity of rubrene measured by ac-calorimetry.” J. Appl. Phys. 114.4 (2013), 043508.

[52] H. Zhang, Y. Yao, Marcia M. Payne, J.E. Anthony, and J.W. Brill. "Thermal diffusivities of functionalized pentacene semiconductors.” Appl. Phys. Lett. 105.7 (2014), 073302.

[53] K. Kurabayashi, M. Asheghi, M. Touzelbaev, and K.E. Goodson. "Measurement of the thermal conductivity anisotropy in polyimide films." J. Microelectromech. Syst. 8.2 (1999), 180-191.

[54] V. Singh et al. "High thermal conductivity of chain-oriented amorphous polythiophene." Nat. Nanotechnol. 9 (2014), 384.

[55] S. Chung, J.-H. Lee, J. Jeong, J.-J. Kim, and Y. Hong, "Substrate thermal conductivity effect on heat dissipation and lifetime improvement of organic lightemitting diodes.” Appl. Phys. Lett. 94 (2009), 253302.

[56] C. Melis, R. Dettori, S. Vandermeulen, and L. Colombo. "Calculating thermal conductivity in a transient conduction regime: theory and implementation." Eur. Phys. J. B 87.96 (2014).

[57] P. Dauber-Osguthorpe, V.A. Roberts, D.J. Osguthorpe, J. Wolff, M. Genest, and A.T. Hagler. "Structure and energetics of ligand binding to proteins: Escherichia coli dihydrofolate reductase-trimethoprim, a drug-receptor system.” Proteins 4.1 (1988), 31-47.

[58] C.I. Bayly, P. Cieplak, W.D. Cornell, and P.A. Kollman. "A well-behaved electrostatic potential based method using charge restraints for determining atomcentered charges: the RESP model.” J. Chem. Phys. 97.40 (1993), 1026910280.

[59] W.D. Cornell, P. Cieplak, C.I. Bayly, and P.A. Kollman. "Application of RESP charges to calculate conformational energies, hydrogen bond energies, and free energies of solvation.” J. Am. Chem. Soc. 115.21 (1993), 9620-9631.

[60] J. Tersoff. "New empirical approach for the structure and energy of covalent systems.” Phys. Rev. B 37.12 (1988), 6991-7000.

[61] Steve Plimpton. "Fast Parallel Algorithms for Short-Range Molecular Dynamics.” J. Comp. Phys. 117.1 (1995), 1-19.

[62] J. Ràfols-Ribé, R. Dettori, P. Ferrando-Villalba, M. Gonzalez-Silveira, L. Abad, A. Lopeandía, L. Colombo, and J. Rodríguez-Viejo. "Evidence of thermal transport anisotropy in stable glasses of vapour deposited organic molecules." Physical Review Materials 2 (2018), 035603.

[63] S.P. Coleman, D.E. Spearot, and L. Capolungo. "Virtual diffraction analysis of Ni $\left[\begin{array}{lll}0 & 1 & 0\end{array}\right]$ symmetric tilt grain boundaries.” Model. Simul. Mater. Sci. Eng. 21.5 (2013), 055020.

[64] C.M. Palumbiny, F. Liu, T.P. Russell, A. Hexemer, C. Wang, and P. MüllerBuschbaum. "The crystallization of PEDOT:PSS Polymeric Electrodes Probed In Situ during Printing.” Adv. Mater. 27.22 (2015), 11-17.

[65] J.F. Franco-Gonzalez and I.V. Zozoulenko. "Molecular dynamics study of mor- 
phology of doped PEDOT: from solution to dry phase.” J. Phys. Chem. B 121.16 (2017), 4299-4307.

[66] X. Wang, J. Zhang, Y. Chen, and P.K.L. Chan. "Molecular dynamics study of thermal transport in a dinaphtho[2, 3 - b : 2', 3' - f]thieno[3, 2 - b] thiophene (DNTT) organic semiconductor." Nanoscale 9.6 (2017), 2262-2271.

[67] A. Gujral, K.A. O'Hara, M.F. Toney, M.L. Chabinyc, and M.D. Ediger. "Structural Characterization of Vapor-Deposited Glasses of an Organic Hole Transport Material with X-ray Scattering.” Chem. Mater. 27.9 (2015), 33413348.

[68] D.M. Leitner. "Thermal Boundary Conductance and Thermal Rectification in Molecules.” J. Phys. Chem. B 117.42 (2013), 12820-12828.

[69] J. Epstein, W.L. Ong, C.J. Bettinger, and J.A. Malen. "Temperature Dependent Thermal Conductivity and Thermal Interface Resistance of Pentacene Thin Films with Varying Morphology.” ACS Appl. Mater. Interfaces 8.29 (2016), 19168-19174.

[70] Y. Jin, S. Nola, K.P. Pipe, and M. Shtein. "Improving thermoelectric efficiency in organic-metal nanocomposites via extra-low thermal boundary conductance." J. Appl. Phys. 114.19 (2013), 194303.

[71] W.G.H. Maraudin. "Theory of Lattice Dynamics in the Harmonic Approximation." (Academic, New York, 1971).

[72] A. Antidormi, X. Cartoixà, and L. Colombo. "Nature of microscopic heat carriers in nanoporous silicon." Phys. Rev. Materials 2 (2018), 056001.

[73] V. Hernandez, J.E. Roman, and V. Vidal, "SLEPc: A scalable and flexible toolkit for the solution of eigenvalue problems.", ACM Trans. Math. Softw. 31 (2005), 351.

[74] S. Balay et al. "PETSc Web page", http://www.mcs.anl.gov/petsc.

[75] S. Balay et al. "PETSc user's manual", Technical Report ANL-95/11, Revision 3.8, Argonne National Laboratory, 2017, URL: http://www.mcs.anl.gov/petsc.

[76] S. Balay, W.D. Gropp, L.C. McInnes, and B.F. Smith, "Efficient management of parallelism in object oriented numerical software libraries.", in Modern Software Tools in Scientific Computing, edited by E. Arge, A.M. Bruaset, and H.P. Langtangen (Birkhäuser Press, Basel, 1997).

[77] R.C. Zeller, R.O. Pohl. "Thermal conductivity and heat capacity of noncrystalline solids." Phys. Rev. B 4 (1971), 2029-2041.

[78] W.A. Phillips. "Amorphous Solids: Low-Temperature Properties. Topics in Current Physics.” (Springer, Berlin, 1981), Vol 24.

[79] W.A. Phillips. "Tunneling states in amorphous solids." J. Low Temp. Phys. 7 (1972), 351-360.

[80] P.W. Anderson, B.I. Halperin, C.M. Varma. "Anomalous low-temperature thermal properties of glasses and spin glasses.” Philos. Mag. 25 (1972), 1-9. 